\title{
Emergence Delirium after Propofol Anaesthesia
}

\section{Letter to the Editor - Case Report}

Ulrich Palm ${ }^{1}$, MD; Johanna Geiger ${ }^{2}$, MD, and Martin Lieb ${ }^{1}$, MD

Short title: Propofol-induced Delirium

${ }^{1}$ Department of Psychiatry and Psychotherapy, Ludwig-Maximilian University, Munich, Germany

${ }^{2}$ Department of Gastroenterology, Ludwig-Maximilian University, Munich, Germany

\section{Address for correspondence:}

Dr. Ulrich Palm

Dept. of Psychiatry and Psychotherapy

Ludwig-Maximilian University

Nussbaumstr. 7

80336 Munich, Germany

Tel.: $+49(0) 89$ 7095-2716

Fax: $+49(0) 897095-5715$

Email: ulrich.palm@med.uni-muenchen.de

Disclosure of financial interests: This work was not supported by any grant or organization.

Word Count Main Text: 615

Number of Tables: $\quad 0$

Number of Figures: $\quad 0$ 


\section{Case report:}

Propofol is a commonly used anaesthetic agent. Recent findings suggest that low-dose propofol sedation reduce the risk of delirium in elderly (1). In children, emergence agitiation was reported (2), even with convulsions, ataxia and hallucinations (3). Besides the rare complication of a propofol infusion syndrome, sexual hallucinations $(4,5)$, epileptic seizures and seizure-like phenomena $(6,7)$ were reported. We report a severe delirious and aggressive state during emergence after propofol anaesthesia.

The 20 year old female patient $(175 \mathrm{~cm}, 75 \mathrm{~kg})$ was passed on a gastroenterological service for nausea and vomiting. She suffered from Morbus Crohn (Enterocolitis regionalis), continuously treated with prednisolone $5 \mathrm{mg}$ and azathioprine $150 \mathrm{mg}$ since three years, adalimumab (TNF-alpha-antagonist) since three months and pantoprazole and tropisetron since two weeks. The past medical history was negative for drug abuse, alcohol or nicotine abuse, metabolic diseases, allergic reactions or treatment with psychiatric drugs. EEG was normal and there was no history of epilepsy or neurological disorder in the patient or among her relatives. Cranial and abdominal MRI was regular. She already had undergone two gastroscopies in propofol anaesthesia without adverse effects.

Anaesthesia was prepared with propofol (1\%, $100 \mathrm{mg}$ bolus, $180 \mathrm{mg}$ continuously over 15 minutes). Sedation was delayed with minimal restlessness. Continuous monitoring showed no relevant hypotonia or hypoxia. During waking up, restlessness increased but the patient was not responsive, eyes were closed. She did not recognize her mother, being present during gastroscopy, and refused body contact by beating, scratching, biting and crying not to touch her. Agitation increased and bed rails had to be attached. The patient received prednisolone $125 \mathrm{mg}$ for coughing fit and midazolam $2 \mathrm{mg}$ for sedation without improvement. An epileptic seizure was deemed unlikely due to the intentioned and coordinated movement pattern and the lacking response to midazolam. Finally, after 25 minutes she received haloperidol $5 \mathrm{mg}$, causing a decline of agitation and aggression within 
a few minutes, followed by one hour of sleep. After waking up, she was fully orientated and reported dreamless sleeping for the period of anaesthesia and complete amnesia for the delirious state. Muscle or bone pain or muscle rigidity did not appear. The patient was observed on normal ward until the following day.

Reports of severe or prolonged agitation are sparse (8); other findings represent the adverse effects of sexual hallucinations or seizure-like phenomena (SLP). The latter is characterized by uncoordinated and tonic-clonic muscle movements $(6,7)$, opisthotonus and athetoid neck and limb movements (9). Signs for SLP or grand-mal epilepsy did not occur in our patient, but she showed intentional, coordinated movements, purposeful defence reaction and anxious affect whereas being unresponsive. Complete amnesia and a dreamless sleep suggest the absence of sexual hallucinations $(4,5)$ despite there probably were delusions and anxiety. The sudden decline of all symptoms after Haloperidol medication supports the diagnosis of a delirium.

It has to be discussed whether the new medication was responsible for the incident. Adalimumab may cause mood changes including depression, anxiety and insomnia (10), but delirium or aggressive behaviour has not been reported yet. Tropisetrone is frequently used in combination with propofol and showed favourable results on nausea and vomiting in this combination (11), due to the similar binding profile at the $5-\mathrm{HT}_{3}$ receptor (12). Adverse effects of this combination are unknown. Pantoprazole may cause depression, hallucinations and disorientation in "predisposed" patients (13), but the patient had no risk factors of neurological or psychiatric disorders. However, the combination of several drugs may have caused this severe adverse effect, but surprisingly after two interventions without side effect, contrarily to the delirium after first time use of propofol (8). In summary adverse effects may occur even if former propofol anaesthesia has been was well tolerated. 


\section{References}

1. Sieber FE, Zakriya KJ, Gottschalk A, et al. (2010) Sedation depth during spinal anesthesia and the development of postoperative delirium in elderly patients undergoing hip fracture repair. Mayo Clin Proc 85:18-26

2. Hasani A, Ozgen S, Baftiu N (2009) Emergence agitation in children after propofol versus halothane anesthesia. Med Sci Monit 15:CR302-CR306

3. Bendiksen A, Larsen LM (1998) Convulsions, ataxia and hallucinations following propofol. Acta Anaesthesiol Scand 42:739-741

4. Balasubramaniam B, Park GR (2003) Sexual hallucinations during and after sedation and anaesthesia. Anaesthesia 58:549-553

5. Marchaisseau V, Molia A, Herlem E, et al (2008) [Propofol-induced hallucinations and dreams]. Therapie 63:141-144

6. Hickey KS, Martin DF, Chuidian FX (2005) Propofol-induced seizure-like phenomena. J Emerg Med 29:447-449

7. Walder B, Tramer MR, Seeck M (2002) Seizure-like phenomena and propofol: a systematic review. Neurology 58:1327-1332

8. Gadalla F, Spencer J (1996) Prolonged delirium after propofol. Can J Anaesth 43:877

9. Sneyd JR (1992) Excitatory events associated with propofol anaesthesia: a review. J R Soc Med 85:288-291

10. Summary of Product Characteristics (2010) Adalimumab. The electronic Medicine Compendium. 
http://www.medicines.org.uk/emc/medicine/21201/SPC/Humira+Pen+and+Syringe/ Accessed 01 September 2010

11. Tramer MR, Walder B (1999) Efficacy and adverse effects of prophylactic antiemetics during patient-controlled analgesia therapy: a quantitative systematic review. Anesth Analg 88:1354-1361

12. Appadu BL, Lambert DG (1996) Interaction of i.v. anaesthetic agents with 5-HT3 receptors. $\mathrm{Br}$ J Anaesth 76:271-273

13. Summary of Product Characteristics (2010) Pantoprazole. The electronic Medicine Compendium.

http://www.medicines.org.uk/EMC/medicine/21688/SPC/Pantoprazole+20mg + gastro -resistant+tablets/ Accessed 01 September 2010 